\title{
El costo de la Diabetes en México
}

\author{
Mexico and the burden of diabetes
}

Eduardo Monterrubio-Angulo ${ }^{1}$, Maria N. Vallejo-Gonzalez ${ }^{2}$, Rebeca M. E. Guzman-Saldaña ${ }^{3}$

\begin{abstract}
:
There are different types of diabetes. Type 2 diabetes accounts for $90 \%$ of cases, caused by factors such as hereditary predis position, environmental factors and lifestyles. This condition affects the quality of life of the patient and their family. The number of this disease has increased, in 2012 there were more than 347 million people affected in the world. And only $25 \%$ maintain adequate medical control.

The economic costs associated with the treatment of diabetes and its complications represent a serious burden for health services and for patients. A prediabetic to receive comprehensive treatment should spend approximately $\$ 93,000 \mathrm{mxn}$ per year, and a diabetic with a complication, almost $\$ 2,000,000 \mathrm{mxn}$ per year. In 2012, diabetes generated worldwide costs of approximately $\$ 471,000$ million, by 2030 this number amounted to 893,000 million dollars.

In 2005 it is estimated that Mexico spent \$ 317 million in costs of care for patients with DM2. 10 ascending in 2011 to 7,000 million dollars.

The direct costs of type 2 diabetes in Mexico were estimated at $\$ 179,495.3$ million in 2013. It should be noted that the cost of medical care for the main complications of diabetes represented the highest percentage (87\%), the indirect costs of type 2 diabetes in Mexico were estimated at $\$ 183,364.49$ million. In this sense, the economic loss due to premature death accounted for most of the burden of indirect costs $(72.5 \%)$.
\end{abstract}

Keywords:

Diabetes, treatment, costs, complications

\section{Resumen:}

Existen diferentes tipos de diabetes. La diabetes tipo 2 representa $90 \%$ de los casos, ocasionada por diversos factores como la predisposición hereditaria, los factores ambientales y de estilos de vida. Este padecimiento afecta la calidad de vida del paciente y sus familiares. La cifra de éste padeciendo ha ido aumentando, en 2012 había más de 347 millones de personas afectadas en el mundo. Y de ellos Solo $25 \%$ mantiene un adecuado control médico.

Los costos económicos asociados al tratamiento de la diabetes y sus complicaciones representan una grave carga para los servicios de salud y para los pacientes. Una persona con prediabetes para recibir tratamiento integral debería gastar aproximadamente $\$ 93000$ pesos al año, y un diabético con una complicación, casi \$2 000000 de pesos al año.

En 2012, la diabetes generó costos a nivel mundial de aproximadamente \$471,000 millones de dólares, para 2030 este número ascenderá a los 893000 millones de dólares. En 2005 se estima que México gasto 317 millones de dólares en costos de atención a pacientes con DM2. ascendiendo en 2011 a 7000 millones de dólares.

Los costos directos de la diabetes tipo 2 en México se estimaron en $\$ 179,495.3$ millones de pesos en el año 2013. Cabe destacar, que el costo de la atención médica de las principales complicaciones de la diabetes representó el mayor porcentaje (87\%) de los costos directos. Por su parte, los costos indirectos de la diabetes tipo 2 en México se estimaron en \$183,364.49 millones de pesos. En este sentido, la pérdida económica por muerte prematura representó la mayor parte de la carga de los costos indirectos $(72.5 \%)$.

Palabras Clave:

\footnotetext{
${ }^{1}$ Autor de Correspondencia. Estudiante del Doctorado en Investigación de la Universidad del Fútbol y Ciencias del Deporte. Pachuca de Soto. México. Email: dr.monterrubio@gmail.com

${ }^{2}$ Estudiante del Doctorado en Investigación de la Universidad del Fútbol y Ciencias del Deporte. Pachuca de Soto. México. Email: n.vallejoglz@gmail.com

${ }^{3}$ Profesora del Doctorado en Investigación de la Universidad del Fútbol y Ciencias del Deporte. Profesora Investigadora del Área Académica de Psicología de la Universidad Autónoma del Estado de Hidalgo. Pachuca de Soto. México. Email: rguzman@uaeh.edu.mx
} 


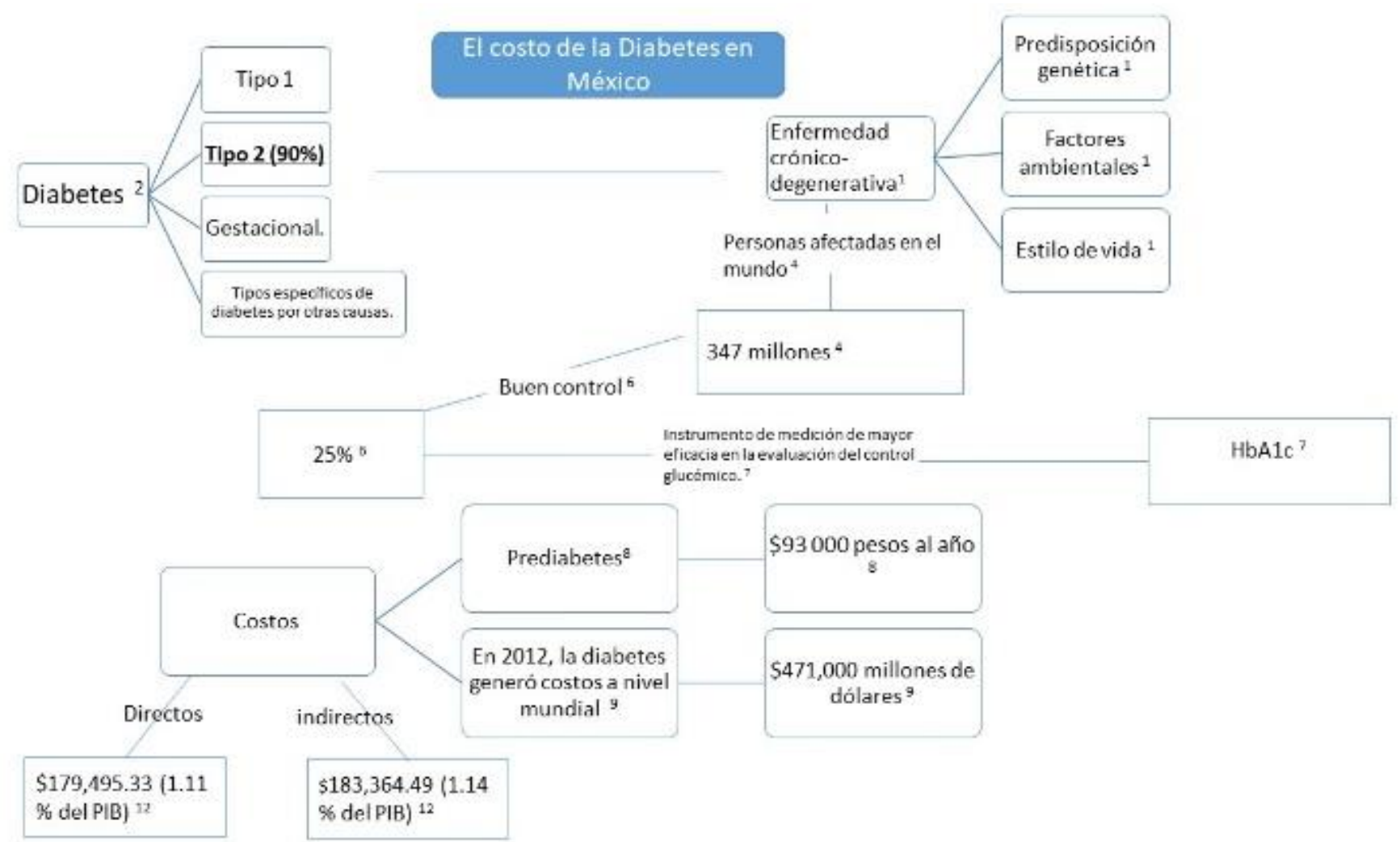

\section{Referencia}

1.- Secretaría de Salud. (1994). NOM-015-SSA2-1994. Recuperado de: http://www.salud.gob.mx/unidades/cdi/nom/015ssa24.html

2.-American Diabetes Association. (2018). Standards of Medical Care In Diabetes 2018. The Journal of clinical and Applied Research and Education, 1-159

3.- Instituto Nacional de Ciencias Médicas y Nutrición Salvador Zubirán (INNSZ). Diabetes Gestacional. Recuperado de: http://www.innsz.mx/ documentos/diabetes/diabetes\%20gestacional\%20smne.pdf

4 .- Organización Mundial de la Salud (OMS). Diabetes. Recuperado de: http://www.who.int/diabetes/action_online/basics/es/index1.html

5.- Federación Internacional de Diabetes (IDF). Atlas. Sexta edición 2014. Mortalidad. Recuperado de: http://www.idf.org/diabetesatlas/5e/es/mortalidad.

6.- Instituto Nacional de Salud Pública (INSP). (2012). Encuesta Nacional de Salud y Nutrición 2012. ENSANUT. Recuperado de: http://ensanut.insp.mx/doctos/seminario/M0302.pdf

7.- Academia Nacional de Medicina (2015). Acciones para enfrentar la diabetes. Documento de postura. Mauricio Hernández Ávila.

8.- Arredondo A et al . Cap 11: Impacto Económico de la Diabetes en México: Retos y Soluciones. Acciones para enfrentar la Diabetes: Documento de postura ANM, 2014: 269-72

9.-Federación Internacional de Diabetes (IDF). (2012). Diabetes Atlas. Recuperado de: http://www.idf.org/diabetesatlas/5e/es/la-carga-mundial?language=es

10.- Federación Internacional de Diabetes (IDF). Atlas. Cuarta edición 2010. Impacto económico de la diabetes. Recuperado de: http://www.idf.org/sites/default/files/Economic\%20impact\%20of\%20Diabetes_0.pdf

11.- Organización para la Cooperación y el Desarrollo Económico (OCDE). (2016). Crecimiento de mortalidad por diabetes mellitus por cada 100 mil habitantes. Recuperado de http://stats.oecd.org/\#

12.- Barraza-Lloréns M, Guajardo-Barrón V, Picó J, García R, Hernández C, Mora F, Athié J, Crable E, Urtiz A (2015) Carga económica de la diabetes mellitus en México, 2013. México, D.F.: Funsalud

13.- Arredondo, A. (2011). Costos de la Diabetes en América Latina: Evidencias del caso Mexicano. Recuperado de: https://www.ispor.org/consortiums/LatinAmerica/documents/ViH/Costos-de-la-Diabetes-en-America-Latina.pdf 